\title{
Management of Pelvic Pain from Dysmenorrhea or Endometriosis
}

\author{
Laeth Nasir, MBBS, and Edward T. Bope, MD
}

\begin{abstract}
Many women suffer from pelvic pain, and a great many visit their family doctor for diagnosis and treatment. Two common causes are primary dysmenorrhea and endometriosis. Primary dysmenorrhea is best treated by prostaglandin inhibition from nonsteroidal anti-inflammatory drugs (NSAIDs) and cyclooxygenase-2 (COX-2)-specific inhibitors. Oral contraceptives can be added to improve pain control. Endometriosis can be treated with NSAIDs and COX-2-specific inhibitors as well but can also be treated with hormonal manipulation or surgery. Empiric treatment for endometriosis in selected patients is now accepted, making the disorder easier for family physicians to manage. (J Am Board Fam Pract 2004;17:\$43-7.)
\end{abstract}

Pelvic pain is one of the most common problems affecting women of reproductive age. The pain may vary from mildly irritating to incapacitating. Dysmenorrhea and endometriosis are the two most common causes. Nonsteroidal anti-inflammatory drugs (NSAIDs) and cyclo-oxygenase-2 (COX-2)specific inhibitors are the mainstays of therapy for both disorders. Hormonal manipulation may also be used in treatment. Surgical and alternative treatments are also discussed.

\section{Definitions}

In the broadest sense, pelvic pain is considered any visceral pain presenting below the umbilicus. This article focuses on the two most common causes of chronic pelvic pain: dysmenorrhea and endometriosis. Pain in the bowel and bladder are considered to be outside the pelvis, although the astute clinician also takes into account the importance of these contiguous organs as causes of pain. Acute pelvic pain is defined as recent in onset, whereas chronic

From the Department of Family Medicine, University of Nebraska at Omaha (LN), and Family Practice Residency Program, Riverside Methodist Hospital, Columbus, OH (ETB). Address correspondence to: Edward T. Bope, MD, ABFP, Riverside Family Practice Residency Program, Riverside Methodist Hospital, 697 Thomas Lane, Columbus, OH 43214 (e-mail: bopee@ohiohealth.com).

The Family Practice Pain Education Project (FP-PEP) acknowledges an unrestricted educational grant from Pfizer to Cardinal Health to produce educational materials for primary care doctors about pain management. The information provided here is the opinions and research of the family physicians who served on FP-PEP.

This work was presented in part at the 2003 American Academy of Family Physicians (AAFP) Scientific Symposium. pelvic pain is that which has lasted greater than 6 months and occurs not solely with menses.

\section{Prevalence}

A number of studies have estimated the prevalence of chronic pelvic pain to be similar to that reported for migraine, low back pain, and asthma. ${ }^{1,2}$ Dysmenorrhea and endometriosis are the two most common causes of pelvic pain. Primary dysmenorrhea is a very common gynecologic problem in menstruating women. Reported prevalence rates are as high as $90 \% ; 1$ in 13 sufferers are incapacitated for 1 to 3 days per month, impacting school and work attendance. Primary dysmenorrhea usually presents during adolescence within 3 years of menses. Most women who suffer from dysmenorrhea do not seek medical care.

Endometriosis is seen in 5 to $10 \%$ of women in the general population and is thought to be more common in the mature woman, but it can also occur in adolescents and has been reported in girls as young as 10.5 years of age. ${ }^{3}$ The peak incidence is between the ages of 25 and 30 years.

\section{Management of Pelvic Pain Dysmenorrhea}

Affected women experience sharp, intermittent spasms associated with their menstrual cycle. It is usually centered in the suprapubic area but may radiate to the back of the legs or the lower back. Systemic symptoms of nausea, vomiting, diarrhea, fatigue, fever, headache, or lightheadedness are fairly common. The pelvic pain of dysmenorrhea has been demonstrated to be mediated through the 


\section{Primary Dysmenorrhea \\ Treatment Algorithm}

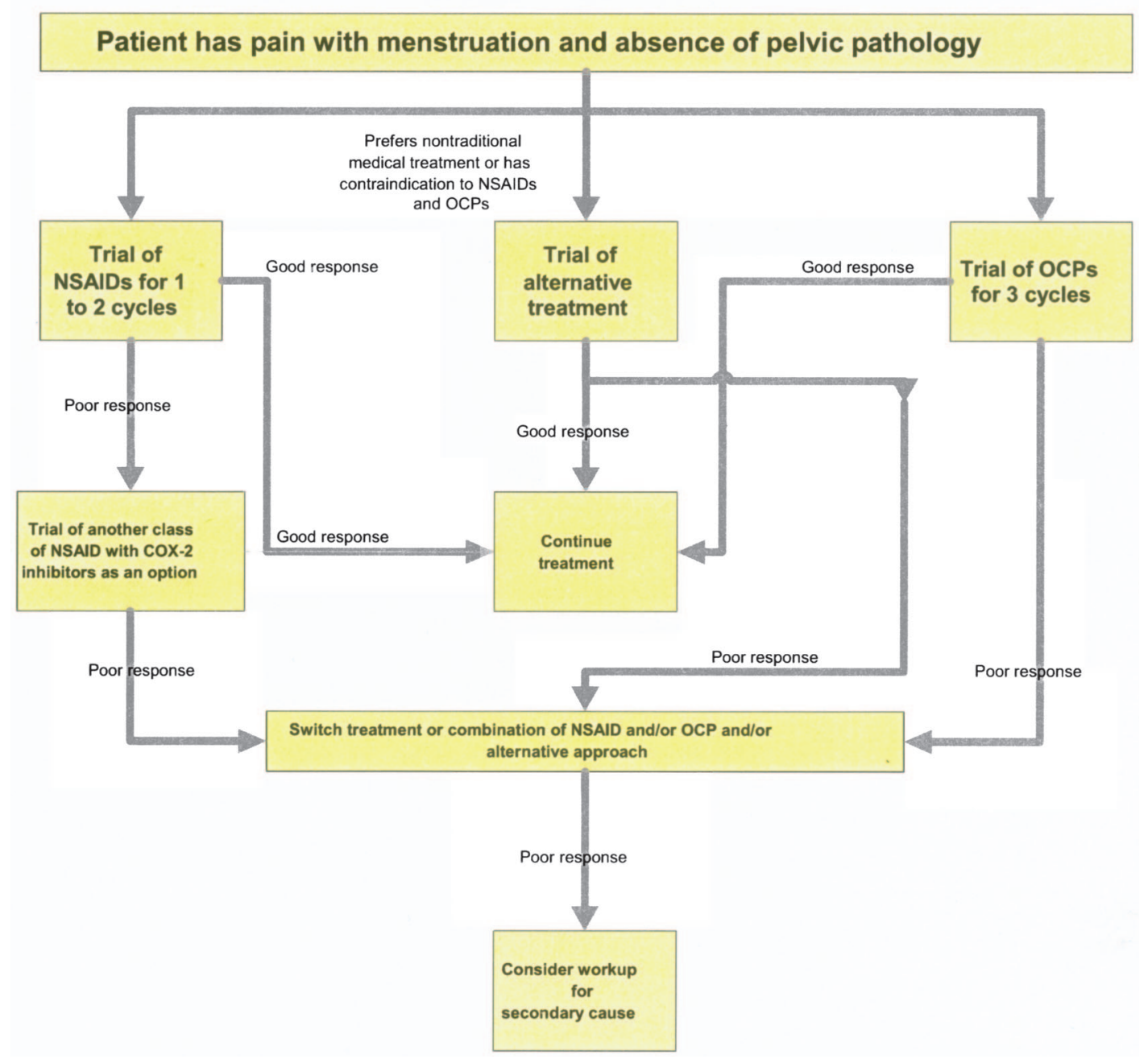

Figure 1. Primary dysmenorrhea treatment algorithm.

action of prostaglandin factor $2 \mathrm{x}$ and is ischemic in nature; therefore, prostaglandin inhibition nearly always diminishes or resolves the pain. Numerous studies have documented the efficacy of standard NSAIDs and COX-2-specific inhibitors acting through prostaglandin synthetase inhibition to control dysmenorrhea (SORT A*). ${ }^{4}$ These medica-

* Levels of evidence using SORT: (1) treatment of pain caused by primary dysmenorrhea with NSAIDs or COX-2specific inhibitors $=\mathrm{A}$; (2) treatment of pain caused by primary dysmenorrhea with oral contraceptive pills = C; (3) treatment of endometriosis empirically = B; and (4) treatment of endometriosis pain with NSAIDs or COX-2specific inhibitors $=\mathrm{C}$. tions are started 1 to 2 days before menses and continued for 2 days after menses starts. This firststep treatment is effective in $80 \%$ of patients. For those who fail to respond, oral contraceptive pills or medroxyprogesterone can be added to achieve control. These medicines are effective in $90 \%$ of patients (SORT C). ${ }^{5}$ Figure 1 presents an algorithm for the management of primary dysmenorrhea.

Some alternative treatments for primary dysmenorrhea have been studied and have shown some success. Topical heat at $38.9^{\circ} \mathrm{C}$ used for 12 hours per day has been found to be as beneficial as ibuprofen. ${ }^{6}$ Four small studies of 126 patients 
Table 1. Secondary Causes of Dysmenorrhea and Chronic Pelvic Pain

Endometriosis

Adenomyosis

Endometrial polyps

Leiomyomata

Pelvic inflammatory disease

Pelvic organ prolapse

Adhesions

Musculoskeletal disorders

Gastrointestinal disorders

Urologic disorders

A history of sexual abuse

showed transcutaneous electrical nerve stimulation (TENS) to give moderate relief in $40 \%$ to $60 \%$ of patients. ${ }^{7,8}$ Acupuncture, when studied in 43 patients for 1 year, showed a $91 \%$ improvement in symptoms and a $41 \%$ decrease in analgesic use. ${ }^{9}$ Daily thiamine $(100 \mathrm{mg})$ for 90 days in 556 patients yielded an $87 \%$ cure rate up to 2 months after treatment ${ }^{10}$ and, in adolescents, daily intake of marine $\Omega-3$ fatty acids netted significant improvement. ${ }^{11}$ Nitroglycerin patches improved pain symptoms in one uncontrolled trial. ${ }^{12}$ These alternative treatments can be used alone or as adjuvants to standard therapy.

If a 3- to 4-month trial of anti-inflammatory, hormonal, or alternative treatments has been ineffective, secondary causes of dysmenorrhea and pelvic pain should be considered (Table 1). In one study of 100 women who had inadequate pain relief with NSAIDs and/or oral contraceptives, almost $80 \%$ had endometriosis on laparoscopy. ${ }^{12}$ Only after these secondary causes have been ruled out would invasive options such as uterosacral nerve ablation, presacral neurectomy, or nerve block procedures, be considered. A Cochrane review did not find sufficient evidence to recommend nerve interruption procedures for the treatment of pelvic pain caused by dysmenorrhea. ${ }^{13}$

\section{Endometriosis}

Endometriosis typically presents with the triad of pelvic pain, dyspareunia, and infertility. Any of these 3 issues could motivate a woman to seek care; most often, pain is the compelling reason for the visit. Endometriosis can be investigated and treated by laparoscopy or can be treated empirically. The traditional approach has been to perform laparos- copy to visually and pathologically make the diagnosis, with the advantage that any endometriosis found can be surgically treated at the same time. In one placebo-controlled, double-blind, randomized trial of women with stage I, II, or III endometriosis, $40 \%$ had alleviation of pain at 6 months that could be attributed to surgical debridement. ${ }^{14}$ Progestin, danazol, or gonadotropin-releasing hormone $(\mathrm{GnRH})$ analogs are generally used postoperatively for greater duration of pain relief (Figure 2). ${ }^{15,16}$

Ling ${ }^{17}$ showed empiric treatment to be effective in low-risk patients, including women aged 18 to 45 years with regular menses, no previous diagnosis of endometriosis, no hormonal treatment in the prior 3 months, no evidence of gastrointestinal or urinary disease, normal pelvic ultrasound, normal complete blood count, normal urinalysis, negative gonorrhea and chlamydia culture, negative human chorionic gonadotropin, and failure of NSAIDs and doxycycline to improve pain symptoms (SORT B). Of patients treated empirically, $80 \%$ experienced significant improvement, including patients without detectable endometriosis at subsequent laparoscopy. The empiric treatment group must be carefully screened to be certain there is no concomitant disease, such as infection or pregnancy.

Whether identified surgically or empirically, endometriosis is treated with one or more of the following: traditional NSAIDs, COX-2-specific inhibitors (SORT C), oral contraceptive pills (OCPs), GnRH agonists, progestins, or danazol. Traditional NSAIDs or COX-2 inhibitors are used initially at maximal or nearly maximal dosage. There is no evidence to support switching from one NSAID to another to improve response, although the practice is frequent. ${ }^{18}$ OCPs are used next if pain relief has not been achieved, and they may be used alone or in combination with NSAIDs. Using the "long cycle" approach with oral contraceptive pills (3 months of pills before a week without pills) can reduce the number of menses, thus improving the quality of life. ${ }^{19}$ No evidence supports switching from one OCP to another to improve response.

High-dose progestins improve endometriosis by deciduation followed by pseudonecrosis and atrophy of lesions. Progestins suppress gonadotropin release and ovarian function; for example, $50 \mathrm{mg} /$ day medroxyprogesterone acetate has been shown to improve symptoms in up to $80 \%$ of patients with endometriosis. ${ }^{19}$ Other regimens for progesterone 


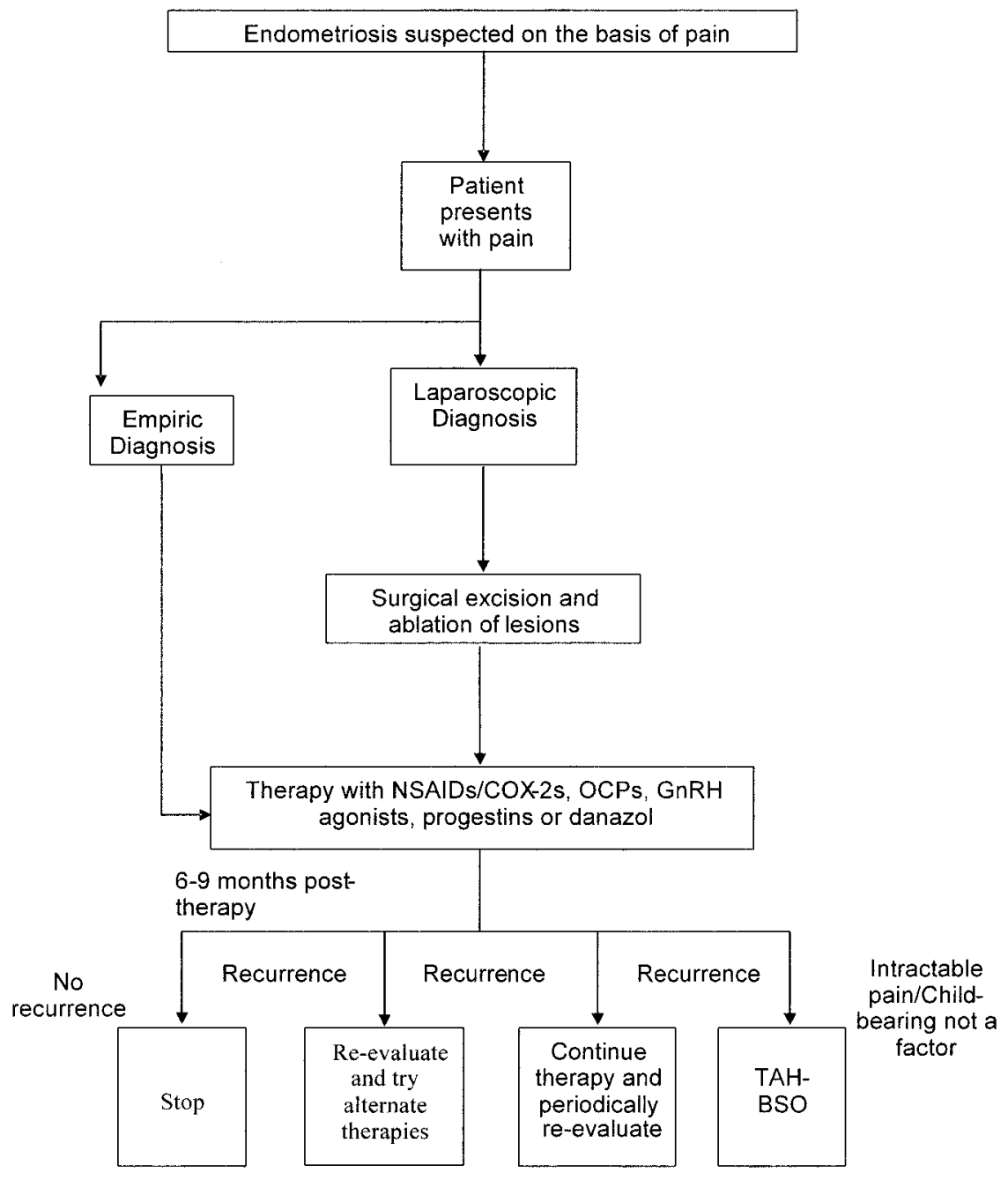

Figure 2. Endometriosis treatment algorithm.

dose and delivery including intramuscular depoprogesterone are effective as well. Side effects of progestin therapy include weight gain, edema, depression, and headache. ${ }^{20}$ Danazol, a testosterone derivative, produces a hypoestrogenic environment and is effective in $80 \%$ of patients; however, the high incidence of androgenic side effects, approaching $80 \%$, limits its use. ${ }^{20}$ The GnRH agonists (eg, nafarelin nasal spray and leuprolide depot), suppress ovarian estrogen production causing estrogen deprivation. The typical course of treatment is for 6 months, after which the patient must be monitored for bone loss and consideration given to adding back estrogen or progesterone. In $50 \%$ of cases, there is recurrence of symptoms within 6 months after GnRH agonist therapy is discontinued. $^{20}$
If empiric treatment or laparoscopy with local ablation has not been successful, then more invasive treatments must be considered, such as uterosacral nerve ablation, presacral neurectomy, or a nerve block procedure. As a last resort, total abdominal hysterectomy and bilateral salpingo-oophorectomy could be considered. ${ }^{21}$

\section{Conclusion}

Evidence supports the use of traditional NSAIDs and the COX-2 specific inhibitors in the treatment of pain associated with primary dysmenorrhea and endometriosis. High levels of effectiveness can be expected for both disorders, meaning that many women could find relief. Although endometriosis treatment has traditionally followed a surgical diagnosis, there is also evidence to support treating 
endometriosis empirically in carefully screened women. Beyond NSAIDs and COX-2-specific inhibitors, there are several medications that may help. Invasive procedures and surgical cures should be reserved for those who are not improved by the algorithms presented here.

\section{References}

1. Zondervan KT, Yudkin PL, Vessey MP, Dawes MG, Barlow DH, Kennedy SH. Prevalence and incidence in primary care of chronic pelvic pain in women: evidence from a national general practice database. BJOG 1999;106:1149-55.

2. Beard RW. Chronic pelvic pain. BJOG 1998;108: $8-10$.

3. Neinstein LS. Adolescent health care: a practical guide, 4th ed. Baltimore: Lippincott Williams \& Wilkins; 2002.

4. Coco AS. Primary dysmenorrhea. Am Fam Physician 1999;60:489-96.

5. Lifford KL. Diagnosis and management of chronic pelvic pain. Urol Clin North Am 2002;29:637-47.

6. Akin MD, Weingand KW, Hengehold DA, Goodale MB, Hinkle RT, Smith RP. Continuous low-level topical heat in the treatment of dysmenorrhea. Obstet Gynecol 2001;97:343-9.

7. Thomas M, Lunden T, Bjork J, LundstromLindsbedt V. Pain and discomfort in primary dysmenorrhea is reduced by pre-emptive acupuncture or low frequency TENS. Eur J Phys Med Rehabil 1995;5:71-6.

8. Dawood MY, Ramos J. Transcutaneous electrical nerve stimulation (TENS) for the treatment of primary dysmenorrhea: a randomized crossover comparison with placebo TENS and ibuprofen. Obstet Gynecol 1990;75:656-60.

9. Helms JM. Acupuncture for the management of primary dysmenorrhea. Obstet Gynecol 1987;69:51-6.

10. Gokhale LB. Curative treatment of primary (spasmodic) dysmenorrhea. Ind J Med Res 1996;103: 227-31.

11. Harel Z, Biro FM, Kottenhahn RK, Rosenthal SL.
Supplementation with omega-3 polyunsaturated fatty acids in the management of dysmenorrhea in adolescents. Am J Obstet Gynecol 1996;174:1335-8.

12. Transdermal nitroglycerine in the management of pain associated with primary dysmenorrhoea: a multinational pilot study. The Transdermal Nitroglycerine/Dysmenorrhoea Study Group. J Int Med Res 1997;25:41-4.

13. Wilson ML, Farquhar CM, Sinclair OJ, Johnson NP. Surgical interruption of pelvic nerve pathways for primary and secondary dysmenorrhoea. Cochrane Database Syst Rev 2000;(2):CD001896.

14. Sutton CJG, Ewen SP, Whitelaw N, Haines P. Prospective, randomized, double-blind trial of laser laparoscopy in the treatment of pelvic pain associated with minimal, mild, and moderate endometriosis. Fertil Steril 1994;62:696-700.

15. Cosson M, Querleu D, Donnez J, et al. Dienogest is as effective as triptorelin in the treatment of endometriosis after laparoscopic surgery: results of a prospective, multicenter, randomized study. Fertil Steril 2002;77:684-92.

16. Vercellini P, Frontino G, De Giorgi O, Aimi G, Zaina B, Crosignani PG. Comparison of a levonorgestrel-releasing intrauterine device versus expectant management after conservative surgery for asymptomatic endometriosis: a pilot study. Fertil Steril 2003;80:305-9.

17. Ling FW. Randomized controlled trial of depot leuprolide in patients with chronic pelvic pain and clinically suspected endometriosis. Pelvic Pain Study Group. Obstet Gynecol 1999;93:51-8.

18. Barbieri RB. Endometriosis. In: Rakel RE, Bope ET, editors. Conn's current therapy, 2002. 54th ed. Philadelphia: WB Saunders; 2002

19. Luciano AA, Turksoy RN, Carleo J. Evaluation of oral medroxyprogesterone acetate in the treatment of endometriosis. Obstet Gynecol 1988;72:323-7.

20. Wellbery C. Diagnosis and treatment of endometriosis. Am Fam Physician 1999;60:1753-68.

21. Carlson KJ, Miller BA, Fowler FJ Jr. The Maine Women's Health Study: I. Outcomes of hysterectomy. Obstet Gynecol 1994;83:556-65. 